Research Paper

\title{
The perception of the Illness with Subsequent Outcome Measure in More Favorable in Continuos Peritoneal Dialysis vs Hemodialysis in the Framework of Appraisal Model of Stress
}

\author{
Zbigniew Nowak $^{1}$, Krzysztof Laudański² ${ }^{凶}$ \\ 1. Department of Nephrology with Dialysis Unit, Military Institute of Medicine, Szaserow 128, Warsaw, Poland \\ 2. Department of Anesthesiology and Critical Care, University of Pennsylvania, Dulles 7, 3400 Spruce St, Philadelphia PA 19146, USA.
}

$\square$ Corresponding author: Department of Anesthesiology and Critical Care, University of Pennsylvania, Dulles 7, 3400 Spruce St, Philadelphia PA 19146. Email: klaudanski@gmail.com

(c) Ivyspring International Publisher. This is an open-access article distributed under the terms of the Creative Commons License (http://creativecommons.org/ licenses/by-nc-nd/3.0/). Reproduction is permitted for personal, noncommercial use, provided that the article is in whole, unmodified, and properly cited.

Received: 2013.01.10; Accepted: 2014.01.01; Published: 2014.02.04

\begin{abstract}
The aim of the study was to use the appraisal model of stress to compare hemodialysis (HD) and continuous peritoneal dialysis (CAPD) patients with special focus on the perception of end-stage renal disease and subsequent emotional profile and health related quality of life (HQoL) in. We hypothesize that different circumstances related to both modes of therapies will result in dissimilar perception of chronic illness with subsequent changes in emotional profile and heath related quality of life. The total of 88 patients with end stage renal disease (ESRD) enrolled in hemodialysis $(n=52 ; H D)$ or continuous peritoneal dialysis $(n=36$; CAPD) were given a battery of psychological tests: The Profile of Mood States, The Nottingham Health Profile, The Stress Situation Assessment Questionnaire, The Social Appreciation Questionnaire and The Situation and Trait and Anxiety Inventory. All patients perceived ESRD in terms of a loss and a threat. Moreover, CAPD patients evaluated ESRD as a challenge. Despite different perception of ESRD no significant difference in the level of fear, anxiety or emotional profile was found. Both HD and CAPD patient were reported more fatiguelinertia and confusion/bewilderment than control groups. The main health related complaints were similar in both ESRD patients with major complaints of sleeping disturbances, motor limitations and lack of energy. From the psychological point of view, CAPD treatment seems more like challenge to the enrolled patient which is positive outcome. Despite different appraisal of stress mood and health related complaints were similar in both groups. This may be a result of optimal regulation of cognitive perception of the stress depending on the circumstances of therapy.
\end{abstract}

Key words: Hemodialysis, continuous peritoneal dialysis, appraisal theory of stress, perception of the disease, mood, health-related quality of life.

\section{Introduction}

The appraisal model of stress integrates stressful stimulus, appraisal of it and coping behavior with subsequent outcomes (emotional, behavioral, psychosomatic) in one conceptual framework [1-3]. Perception of disease is an obligatory and initial part of a patient's cognitive assessment of every stressful situation but the appraisal model of stress is underserving underappreciated in medicine. Cognitive appraisal is step-wise process. Primary appraisal is initiated after a stressful stimulus challenges the patient's status quo. 
If the stimulus is perceived as a benign or neutral one, it will be ignored or even appreciated. If the stimulus is deemed stressful or dangerous, it will be categorized as a loss, challenge or threat. Coping process differ between individuals according to each person's predisposition towards certain stress assessment and coping strategies [3]. Alteration in emotional status will follow certain coping strategies depending how stress is perceived. [1-3]. More specifically, an evaluation of the disease as a loss leads to a more negative emotional status whereas perceiving the disease as a challenge may improve mood. The idea of challenge carries a hope for the future and may inspire the patients to an increased effort to cope with the illness. Threat is directly related to an increased anxiety level. Anxiety can paralyze a patient's behavioral efforts to transform a stressful situation into a more agreeable one, with a reduction/augmentation in his/her adherence to treatment. High anxiety is often used as a measure of treatment intrusiveness and the relative effectiveness of the subject's coping strategies. The adequacy of an individual's coping strategy is evaluated in the secondary appraisal where in an individual places the primary appraisal of stress into the context of his/her own expectations and well-being $[2,3]$. In a recent meta-analysis, perception of disease was shown to be one of the most important factors determining the health-related quality of life in dialysed patients [4].

End-stage renal disease (ESRD) is most frequently treated with renal replacement therapy (RTT) $[5,6]$. This therapy imposes severe stress on the patients, which is related to the severe diet restriction, extensive drug regimen, and strict dialysis schedule $[4,6,7]$. RTT can be performed in two main ways. Hemodialysis (HD) relies on the patient visiting a specialized center 2-3 times per week for 4-6 hours. During this procedure, the patient rests while an automated process removes metabolic products from the blood. The procedure requires a patient's arterio-venous fistula to be accessed with a large bore needle each time it is performed. Alternatively, continuous ambulatory peritoneal dialysis (CAPD) can be implemented. In this treatment, the dialysis fluid is exchanged via per-implanted abdominal catheter using patient's peritoneum as dialysis membrane. The entire procedure can be done by patients in-house. Even this brief description of the two different RTT treatments suggests there are different psychological challenges and stressors in each case. These stressors will interact with the patient's pre-determined values and appraisal schemes, ultimately affecting the patient's mood and sense of well-being. In turn, if this process is too much of a burden to the patient, he/she may modify it in order to lower the stress level [8].
Previous studies examined the mood, frequency of depression, some coping strategies in dialyzed patients, but did not use a conceptual framework to analyze the chain of circumstances of therapy, appraisal of stress and subsequent mood and self-reported level of somatic complaints $[4,6,7]$. According to the current theories of stress, all of the factors discussed above are related to each other. So describing the psychological process in dialyzed patient in the framework of specific theory exemplifies patient's specific psychological context within the realm of predicted behavior [1-3, 8]. Nevertheless, patients undergoing dialysis were rarely studied this way or only few dimension of their psychological well-being were investigated. Also, we focused on mood which is long-term emotional state while other were interested in emotional status at given moment.

We hypothesized that the diverse circumstances related to enrolment ESRD patients in two alternatives of renal replacement therapies (RRT), hemodialysis (HD) and continuous ambulatory peritoneal dialysis (CAPD), may result in different perception of ESRD with preliminary assumption that CAPD is related to more favorable appraisal. More favorable outcome should result in advantageous mood state and subsequent better perception of somatic health. Thus, we decide to measure three different ways to assess ESRD stress and related outcome in order to determine which mode of dialysis is preferable in the patient's psychosomatic realm.

\section{Materials and Methods}

The study population consisted of chronic in-center hemodialysed patients $(n=52)$, and patients enrolled in an in-home CAPD program $(n=36)$. To ensure that the patients were settled into the routine of dialysis, the interviews were performed at least 3 months after the onset of RRT. Patients below the age of 18 and patients with serious co-morbid illness were excluded from the study. To validate the findings of the study, a healthy control group (CONTR; $n=88$ ) was asked to imagine being in the state of acute illness. Since this group is exposed to different kind of stress (acute and imaginative $v$ s acute and real) we would expect different kind of response thus validating our study.

Mean potassium, urea, creatinine, total protein and hematocrit levels were recorded one week before the interview as a measure of the patients' clinical well-being. The CONTR group consisted of healthy volunteers matched to the patients by age, sex, educational level and employment. CAPD interviews took place during hospitalization for a routine check-up whereas HD patients were examined while undergoing scheduled dialysis. The controls were 
interviewed at their work or home. After explaining the aim of the study, the examinee was asked to fill out a battery of questionnaires according to the attached instructions. A psychologist supervised each subject throughout the procedure.

All participants gave written informed consent to be part of the study. The Ethical Committee at the Department of Psychology of the Warsaw University approved the research protocol.

Anxiety level and perception of disease were measured by The Cognitive Situation Appreciation Questionnaire (CSAQ) and The State-Trait Anxiety Inventory (STAI) respectively [9]. Both surveys consist of two parts. Part B is the measure of the inherited predisposition to react stressfully (STAI) or to see all stressful events in a loss, threat or challenge (CSAQ). The second parts of the STAI and CSAQ are design to be modified depending on the purpose of the study so that the dialyzed subjects were asked about ESRD whereas the healthy volunteers were asked to imagine facing an acute, transient illness (e.g. flu). STAI is a direct score of the anxiety level, but CSAQ has three independent scales to measure the perception of stressful events: loss, challenge and threat. Mood was evaluated by The Profile of Mood States (POMS) [10], which is the WHO-recommended questionnaire for studying emotional outcomes of various medical therapies [11]. It describes mood in six dimensions: Tension/Anxiety, Depression/Dejection, Anger/Hostility, Vigor/Activity, Fatigue/Inertia and Confusion/Bewilderment. POMS has the advantage of measuring mood defined as semi-stable emotional profile over longer period of time. To measure HRQoL The Nottingham Health Profile (NHP) was employed [12]. It measures an individual's perception of his/her health status on six subscales: energy, pain, sleep, emotional reactions, mobility limitations and social isolation. The Social Appreciation Questionnaire (SAQ) was used to measure subjects' propensity to answer the investigator's question the way he/she thinks is expected by researcher (conformity) [13]. By correlating the results from CSAQ with the other variables we were able to statistically control for the subjects' conformity on the other measured variables [14]. Polish adaptations of the original surveys have satisfactory psychometric characteristics [2, 9, 12, 14, 15]. CSAQ, POMS, NHP and STAI have been previously employed, and are well established tools [3, 10, 11].

Psychological, clinical and demographic variables are presented as mean $(\mathrm{X})$ and standard deviation (SD). Decisions regarding the use of parametric versus nonparametric tests were based on the Kolmogorov-Smirnov and Levene statistics for determining the normality and the equality of group variance, respectively. One-way analysis of variance (ANOVA) with post-hoc LSD test and Student $t$-test was performed for parametric data, whereas H-Kruskal-Willis and U Mann-Whitney tests were performed for comparisons of nonparametric variables. The correlation momentum was computed as a $r$-Pearson. Statistical significance was set at a 2-tailed P<0.05. SPSS v8.0 (Tulusa, AZ) software was used for all computations. $11,3 \%$ of the questionnaire were not filling in due to the subject's neglect.

\section{Results}

In first step we compare the demographic and clinical variables to assure that studied groups were comparable. There was no difference in age or gender between the HD, CAPD and CONTR populations, but more subjects were unemployed in the patients' groups than in the controls (data not shown). In order to evaluate the effect of the demographical variables on the data, several correlations were done. Only employment status was related to higher scores on dimensions of: situation-anxiety; perception of ESRD as a challenge; a predisposition to perceive the stress in general as a challenge, a loss and a threat; and a higher intensity of depression/dejection and fatigue/inertia emotions. A one-way analysis of co-variance (ANCOVA) was computed where appropriate. There was no significant correlation between scores on SAQ and other measured variables (data not shown), indicating that subjects' conformity did not biased surveys results.

Since patients' psychological well-being can be affected by inadequacy of dialysis we checked medical characteristic between studied groups. Among dialyzed individuals with $\mathrm{HD}$ or CAPD, there were no differences in the duration of kidney disease $(\mathrm{HD}=11.2 \pm 9.9$; $\mathrm{CAPD}=10.3 \pm 8.1$ [yrs]), duration of dialysis $(\mathrm{HD}=2.1 \pm 2.8 ; \mathrm{CAPD}=2.3 \pm 2.7$ [yrs] $)$, hematocrit level $\quad(\mathrm{HD}=30.5 \pm 2.7 ; \quad \mathrm{CAPD}=30.5 \pm 3.5[\%]), \quad$ urea $(\mathrm{HD}=139.1 \pm 29.8 ; \mathrm{CAPD}=126.6 \pm 31[\mathrm{mg} / \mathrm{dl}])$, creatinine $(\mathrm{HD}=9.8 \pm 1.7 ; \mathrm{CAPD}=10.4 \pm 2.7[\mathrm{mg} / \mathrm{dl}])$, total protein $(\mathrm{HD}=6.4 \pm 1.1 ; \mathrm{CAPD}=6.4 \pm 0.3[\mathrm{mg} / \mathrm{dl}])$ and alkaline phosphatase $\quad(\mathrm{HD}=83.9 \pm 30.8 ; \quad \mathrm{CAPD}=97.7 \pm 62.6$ $[\mathrm{IU} / \mathrm{ml}])$. The potassium level was significantly higher $(\mathrm{HD}=5.3 \pm 0.6$ vs. $\mathrm{CAPD}=4.6 \pm 0.7 ; \mathrm{p}<0.05)$ in the HD group. This may be an artifact related to the mode of RRT. Serum potassium level has not been reported to affect psychological variables; thus no ANCOVA was calculated.

\section{Psychological assessments}

Finally, we studied our primary hypothesize by investigating perception of ESRD-stress and RTT with subsequent emotional and somatic profile. Patients in both HD and CAPD groups judged ESRD as a threat and loss more often than subjects from control group. The intensity of this assessment was similar in both 
the HD and CAPD group (Fig. 1). However, patients being treated with CAPD had a much a higher appraisal of ESRD as a challenge compared to both the HD patients and the healthy volunteers. There was non-significant difference in subjects' inherited predisposition to perceive stressful situations any of three measured perceptions (loss, threat, challenge) as measured by the CSAQ (data not shown). Like wisely, there were also no statistically significant differences in trait-anxiety or illness-related anxiety between the HD, CAPD and CONTR groups (data no shown).

Several subscales from the POMS were significantly different between the groups (Fig.2). The main effects were present in dimensions of fatigue/inertia $(\mathrm{F}[3,136]=4.87 ; \mathrm{p}<0.05)$, confusion/bewilderment $(\mathrm{F}[3,136$ ] $=15.7 ; \mathrm{p}<0,01)$ and depression/dejection $(\mathrm{F}[3,136]=8.58$; $\mathrm{p}<0.01)$. Post hoc analysis revealed that both groups of patients in general are more depressed, tired and confused than CONTR. The intensity of these emotions was similar in both patients groups. However, the significance of depression/dejection was negligible when we controlled for hemoglobin concentration $(\mathrm{F}[3,135]=2.01 ; \mathrm{p}=\mathrm{ns})$. Other subscales of POMS were comparable between groups.

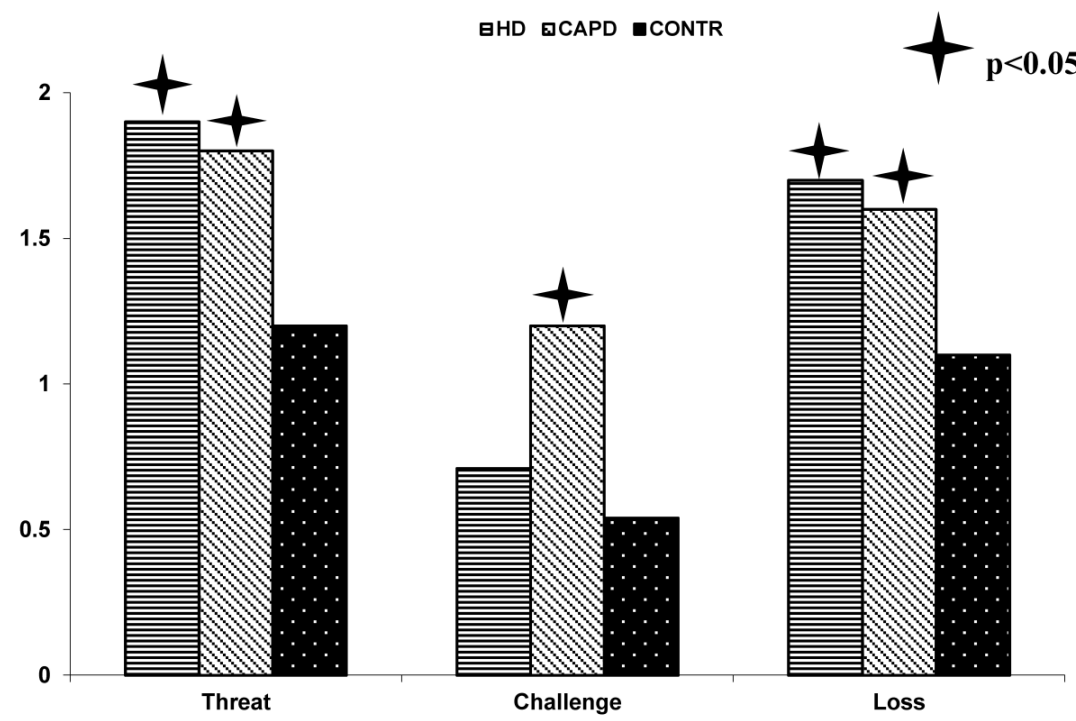

Figure I. Perception of ESRD-related stress in HD, CAPD and CONTR groups.

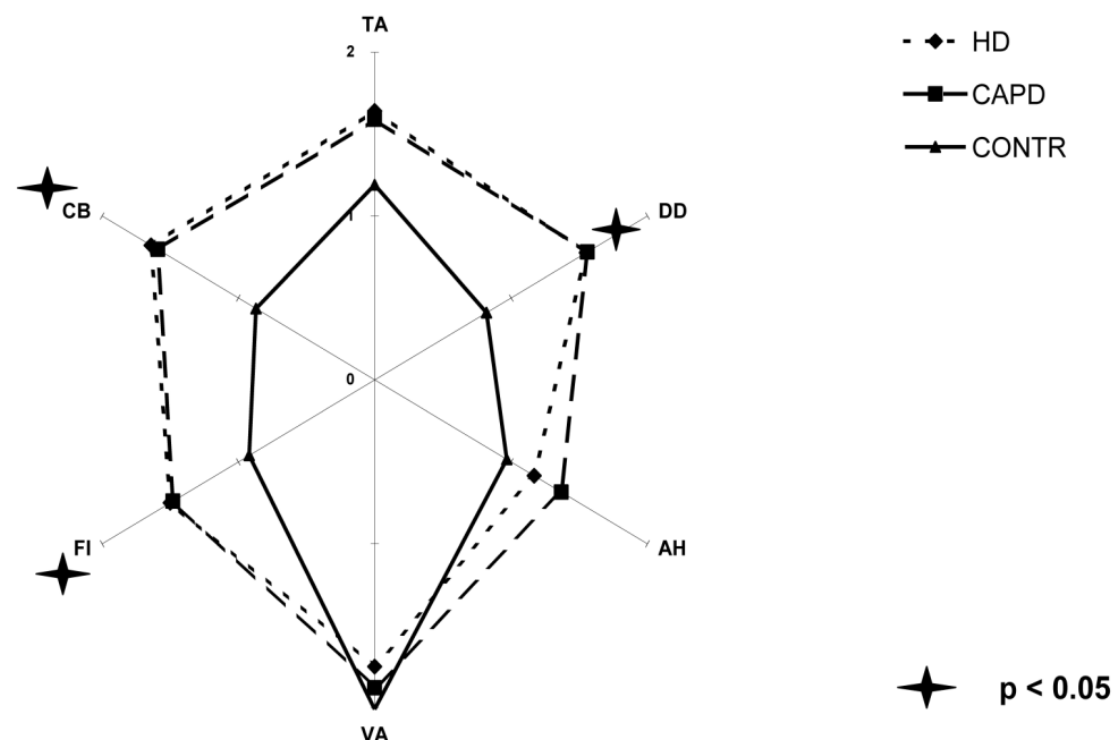

Figure 2. Dimensions of mood state among groups (TA - tension/anxiety, DD - depression/dejection, AH - anger/hostility, VA - vigor/activity, FI fatigue/inertia, $C B$ - confusion/bewilderment). 
GHD ECAPD ICONTR

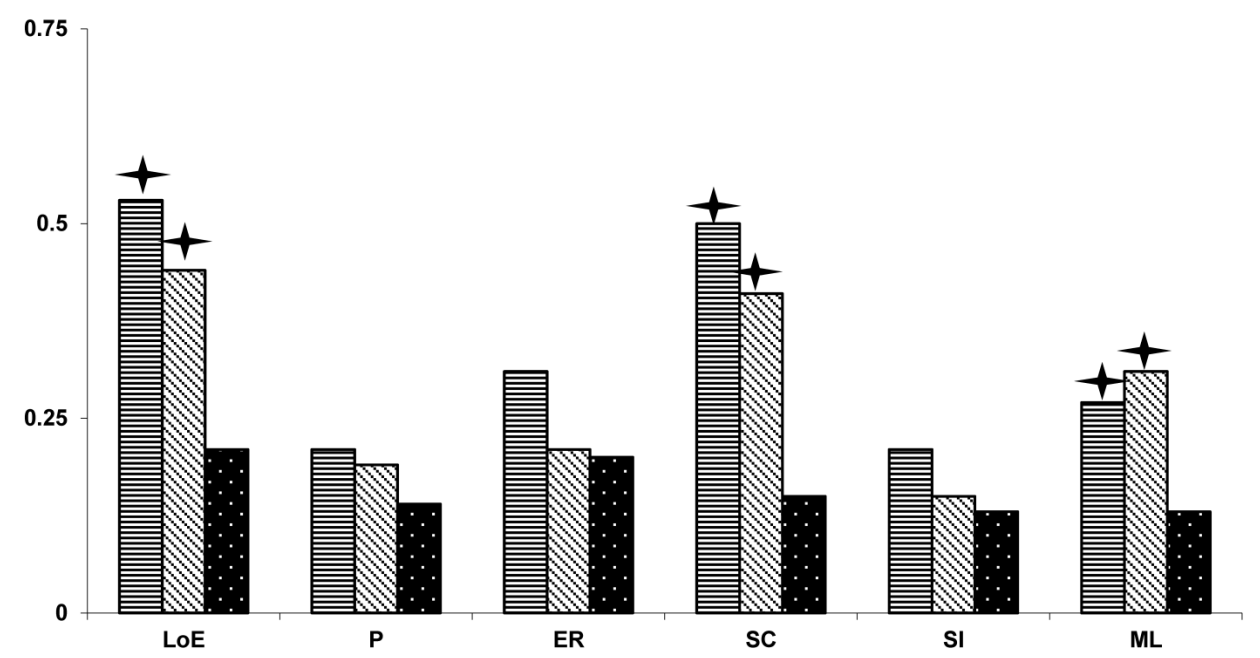

Figure 3. Health related complaints in all groups (LoE - lack of energy, $\mathrm{P}$ - pain, ER - emotional reaction, $\mathrm{SC}$ - sleep complains, $\mathrm{SI}-$ social isolation, $\mathrm{ML}$ - motor limitation).

Because the cognitive appraisal of disease may lead to a certain emotional status, a correlation matrix was computed between one's predisposition to assess ESRD-related disease perception and emotional profile. In HD cognitive appraisal of ESRD as a threat correlated with tension/anxiety $(\mathrm{r}=0.34 ; \mathrm{p}<0.01)$, depression/dejection $(\mathrm{r}=0.32 ; \quad \mathrm{p} 0<0.01)$ and confusion/bewilderment $(\mathrm{r}=0.41 ; \mathrm{P}<0.01)$. This last emotion was also significantly correlated with the perception of ESRD as a loss $(r=0.41 ; \mathrm{p}<0.01)$. CAPD patients perceiving the disease as a threat correlated with tension/anxiety $(\mathrm{r}=0.46 ; \mathrm{p}<0.01)$, fatigue/inertia $(\mathrm{r}=0.44$; $\mathrm{p}<0.01)$ and confusion/bewilderment $(\mathrm{r}=0.52 ; \mathrm{p}<0.01)$. In controls, a threat was related to tension/anxiety $(\mathrm{r}=0.56$; $\mathrm{p}<0.01)$, depression/dejection $(\mathrm{r}=0.45 ; \mathrm{p}<0.01)$, anger/hostility $(\mathrm{r}=0.48 ; \mathrm{p}<0.01)$ and fatigue/inertia $(\mathrm{r}=0.45$; $\mathrm{p}<0.01)$. A challenge was related to vigor/activity (0.46; $\mathrm{p}<0.01)$ in controls, whereas a loss correlated with confusion/bewilderment $(\mathrm{r}=0.36 ; \mathrm{p}<0.01)$.

Several differences in NHP scores were revealed while comparing the HRQoL evaluation within the studied groups. The HD patients over 50 years old reported higher pain complaints $(\mathrm{z}[48]=4.56 ; \mathrm{p}<0.01)$ and mobility limitations $(\mathrm{z}[46]=4.89 ; \mathrm{p}<0.01)$ vs. younger HD patients (age less than 50). Conversely, these differences were not observed in either the CAPD or the CONTR group. Sex and employment status did not affect NHP scores in any group. In both ESRD patients' RTT group main complaints were: lack of energy $(\mathrm{F}[2,104]=8,183 ; \quad \mathrm{p}<0,01)$, sleep disturbances $(\mathrm{F}[2,104]=11,962 ; \mathrm{p}<0,01)$ and mobility limitations $(F[2,104]=4,759 ; p<0,05)$ (Fig. 3). No differences were shown when comparing these variables between HD and CAPD patients. The correlation matrix did not reveal significant links between perception of disease and patients' health complaints.

\section{Discussion}

This study describes the perception of ESRD in two dialyzed populations within the framework of appraisal theory of stress in order to assess the dynamic influence of the components in a holistic way. More specifically, we hypothesize that CAPD is going to result in different perception of ESRD-stress with subsequent more favorable mood profile as compared to the patients treated with hemodialysis. We found that, the dialyzed patients perceive their disease as a threat and a loss, which is a common approach to chronic disease. Moreover, CAPD patients have a pronounced perception of ESRD as a challenge. The observed difference is probably related to the specificity of circumstances of RRT: The CAPD patients are less hospital dependent and solely responsible for their dialysis treatment; they have a higher degree of autonomy than the HD group; and they usually have a higher degree of acceptance to treatment. CAPD patients must completely reorganize their life, but this form of RRT allows for a much greater degree of autonomy and freedom than patients with HD [5]. Consequently, they have a better chance to actively enjoy everyday activities. It seems that this is more beneficial to the patients seems put them in more active role as oppose to perception of the diseases as a loss or threat. This may result in the perception of the disease as a challenge.

In contrast, $\mathrm{HD}$ is based on regular visits in the dialysis unit. For HD recipients, it is much harder to figure out an independent way of life [5]. Moreover, several authors pointed out that this group is older and more passive $[4,7]$. Also, undergoing hemodial- 
ysis is a passive process for patients. This may result in lower anxiety level and lack of perceived need to get more actively involved in the treatment. Thus, these patients are not willing to confront ESRD-related stress.

Cognitive appraisal is a first step in coping with a stressful situation and can affect the subsequent emotional profile [1]. Therefore, we investigated the emotional profile in the studied groups. We speculate that due to the circumstances of treatment and appraisal of the illness-related stress CAPD may be related to higher level of anxiety while patient treated with HD will be more depressed and withdrawn. Surprisingly, we found no differences in the level of anxiety between patients treated with CAPD and HD. We excluded several clinical parameters affecting the anxiety and emotional profile such as: age, trait-anxiety and the pressure to please the investigator (conformity), as factors affecting anxiety scores. Also, clinical and demographical variables were no different between patients treated with two different modes of RTT. Alternatively, the patient's anxiety level is affected by his/her cognitive and behavioral efforts to cope with the distress $[1,3,6,11]$. We hypothesize that although both CAPD and HD patients have different stressful events, the coping strategies employed by these patients are sufficient to maintain anxiety at a normal level, indistinguishable from that of general population. Due to the different circumstances related to the two modes of RTT, patients have to employ different coping strategies. This may explain why the patients' anxiety level is similar, despite an objectively different treatment-related stressor.

Despite the ability to effectively cope with anxiety, several emotions are more intense in the dialyzed population. Fatigue, inertia, confusion and bewilderment seem to be typical for dialyzed patients. Depression level correlated to the anemia, which is well known fact but hemoglobin level was similar in both groups of dialyzed patients [16]. But other emotions were found to affect the perception of ESRD as a threat. Prolonged coping with negative emotions may contribute to the feeling of being "burned out" $[2,4,8$, 9]. The dependence on treatment may also lead to the feeling of being stigmatized by the disease. These emotions are amplified by the perception of the situation as a loss and threat, resulting in a positive feedback loop. All these effects are observed in our study. They are partly related to the general disapproval of being ill.

According to the transactional theory of stress, the perception of the disease may affect the general perception of health status $[2,3]$. We compared the dialyzed patients to demographically matched peers. Therefore, we were able to evaluate the difference between the ESRD patients and the general population. We found that the patients' main health problems are related to lack of energy, sleep disturbance and motor limitations. Lack of energy is directly related to the depressive mood, fatigue and inertia [7, 10, 12]. Motor limitations may reflect problems with the musculoskeletal system. However, this may be an artifact caused by the relatively older population studied in our research program. Sleep disturbances are the most frequently reported problem of patients with ESRD [5]. All of these complaints are much more pronounced when compared to the age-matched population of individuals without serious disease. Interestingly, in our study we could not show a strong relationship between HRQoL and cognitive appraisal of ESRD.

\section{Conclusions}

In summary we conclude that from the psychological point of view, CAPD is favored over HD because it is related to a better perception of ESRD. However, contrary to our original hypothesize different perception of the ESRD-related stress did not lead to dissimilar emotional profile or level of somatic complaints between patients treated with hemodialysis or CAPD. Both emotional profile and profile of somatic complaints in both groups of patients with ESRD was similar as reported previously [5]. Our results suggest that employment of specific coping mechanisms may be responsible for reported lack of difference in the mood and HRQoL.

\section{Acknowledgments}

The authors of this manuscript would like to thank Kazimierz Wrześniewski for his help in conducting this study.

\section{Competing Interests}

The authors have declared that no competing interest exists.

\section{References}

1. Taylor SE. Health Psychology. New York, USA: McGraw Hill Higher Education. 2008

2. Wrzesniewski K., Wlodarczyk D. The role of cognitive appraisal in coping with myocardial infarction: selected theoretical and practical models. Pol Psych Journal 2001; 32: 18-26.

3. Lazarus RS. Toward better research on stress and coping. Am Psychol. 2000; 55: 665-673

4. Chan R, Steel Z, Brooks R, Heung T, Erlich J, Chow J, et al. Psychosocial risk and protective factors for depression in the dialysis population: a systematic review and meta-regression analysis. Journal of Psychosomatic Research. 2011; 71: 300-310.

5. Laudanski K, Nowak Z, Wankowicz Z. Psychological aspects of dialysis: does cognitive appraisal determine the overall outcome. Pol Arch Med Wewn 2010; 120: 49-52.

6. Christensen AJ and Ehlers SL. Psychological factors in end-stage renal disease: an emerging context for behavioral medicine research. J Consult Clin Psychol. 2002; 70: 712-724.

7. Yeh SC and Chou HC. Coping strategies and stressors in patients with hemodialysis. Psychosomatic Medicine. 2007; 69: 182-190. 
8. Gillanders S, Wild M, Deighan C, Gillanders D. Emotion regulation, affect, psychosocial functioning, and well-being in hemodialysis patients. Am J Kidney Dis. 2008; 51: 651-662

9. Wrzesniewski K, Sosnowski T. Situation and trait anxiety inventory - polish adaptation. Warsaw: Wydawnictwo Psychologiczne Uniwersytetu Warszawskiego. 1987.

10. Hunt SM, McEwen J and McKenna SP. Measuring health status. London, GB: Croom Helm; Dover N.H. 1986

11. McNair DM, Lorr M and Droppleman LF. Profile of mood states manual. San Diego, CA: Educational\&Industrial Testing Service. 1992.

12. Wrzesniewski K. Polish Adaptation of Nothingham Health Profile. In: Karski JB and Kirschner H, ed. Current options for health measurment. Warsaw: Wydawnictwo IGP. 1997: 37-42.

13. Drwal B, Wilczynska J. Social Appreciation Questionnaire. Przegl Psych. 1987; 31: 753-761.

14. Dudek B, Koniarek J. Polska adaptacja POMS. Przegl Psych. 1987:753-761.

15. Hambleton RK, Merenda PF, Spielberger CD. Adapting educational and psychological tests for cross-cultural assessment. Mahwah N.J.: L Erlbaum Associates; 2005

16. Li S, Foley RN, Collins AJ. Anemia and cardiovascular disease, hospitalization, end stage renal disease, and death in older patients with chronic kidney disease. Int Urol Nephrol. 2005; 37: 395-402. 